\title{
PLANE MODELS FOR RIEMANN SURFACES ADMITTING CERTAIN HALF-CANONICAL LINEAR SERIES. II
}

BY

ROBERT D. M. ACCOLA ${ }^{1}$

\begin{abstract}
For $r>2$, closed Riemann surfaces of genus $3 r+2$ admitting two simple half-canonical linear series $g_{3 r+1}^{r}, h_{3 r+1}^{r}$ are characterized by the existence of certain plane models of degree $2 r+3$ where the linear series are apparent. The plane curves have $r-23$-fold singularities, one $(2 r-1)$-fold singularity $Q$, and two other double points (typically tacnodes) whose tangents pass through $Q$. The lines through $Q$ cut out a $g_{4}^{1}$ which is unique. The case where the $g_{4}^{1}$ is the set of orbits of a noncyclic group of automorphisms of order four is characterized by the existence of $3 r+3$ pairs of half-canonical linear series of dimension $r-1$, where the sum of the two linear series in any pair is linearly equivalent to $g_{3 r+1}^{r}+h_{3 r+1}^{r}$.
\end{abstract}

1. Introduction. By Torelli's theorem the conformal type of a closed Riemann surface is determined by the symplectic equivalence class of its period matrices. Consequently, special properties that a Riemann surface might possess should be reflected in its period matrix. An attractive approach to this problem is to examine the properties of the theta function especially when evaluated at points of finite order on the Jacobian since quite often these properties are independent of the particular period matrix at hand. Thus Riemann characterized hyperelliptic Riemann surfaces of genus three by the theta function vanishing to order two at one half-period [13], and Weber analogously characterized hyperelliptic Riemann surfaces of genus four [15]. Martens completed the program of characterizing hyperelliptic Riemann surfaces in terms of vanishing properties of theta functions at half-periods [12]. It seems clear that further hyperelliptic automorphism groups can be characterized by the vanishing of certain homogeneous polynomial expressions in theta-nulls, although the author does not know if this program has been carried out.

An extension of this work was begun by Farkas when he discovered extraordinary vanishing properties at half-periods for the theta functions for Riemann surfaces admitting fixed-point-free automorphisms of order two [10]. Following this work of Martens and Farkas the present author was able to characterize Riemann surfaces admitting other automorphisms of period two (involutions) in terms of vanishing properties of the theta function [4].

In another direction, it has long been known that a Riemann surface of genus six

Received by the editors October 9, 1979.

1980 Mathematics Subject Classification. Primary 30F10; Secondary 14 H99.

Key words and phrases. Riemann surface, linear series, algebraic curve, automorphism.

${ }^{1}$ Research supported by the National Science Foundation. The author thanks Professor Joseph Harris for valuable discussions concerning this paper. 
whose theta function vanishes at a single half-period to order three admits a plane model as a nonsingular quintic. In Part $I$ of this paper the author extended this type of result to Riemann surfaces of genus $3 r$ where the theta function vanishes to order $r+1$ at one half-period [6]. The present work is an extension of this latter kind of result.

Kraus seems to be the first author to investigate plane curves where the corresponding theta function vanishes at half-periods [11]. Here he used Riemann's solution to the Jacobi inversion problem which asserts, among many other things, a one-to-one correspondence between the vanishing of the theta function at half-periods to order $r+1$ and the existence of complete half-canonical linear series of dimensions $r, g_{p-1}^{r}$ 's. Kraus constructed plane curves with fairly obvious halfcanonical linear series. The present author continued this work (no intermediate work is known) and constructed plane curves of genus $3 r, 3 r+2$, and $3 r+3$ admitting respectively one, two and four simple half-canonical linear series of dimension $r$. (In the last case the sum of the four linear series is bicanonical [2].)

These two kinds of investigations concerning theta functions, characterizing automorphism groups and certain plane models, are not unrelated. For if a Riemann surface has genus less than $3 r$ and the theta-function vanishes at a half-period to order $r+1$ then this implies the existence of an involution. Similarly, surfaces of genus less than $3 r+2$ or $3 r+3$ admitting respectively two or four half-canonical series of dimension $r$ also admit involutions [4].

To the author's surprise and pleasure the indicated vanishing properties for the theta function for Riemann surfaces of genus precisely $3 r, 3 r+2$, and $3 r+3$ usually characterize the existence of the plane models discussed in [2]. This allows one to compute the dimension in Teichmüller space of surfaces admitting such vanishing properties since by classical methods it is easy to compute the dimensions of these families of plane curves.

Basic to these investigations is the classical work of Castelnuovo on the inequality that bears his name [7], [8]. In almost all these cases the simple half-canonical $g_{p-1}^{r}$ determines a curve in $P^{r}$ that lies on a rational normal scroll whose rulings cut out a $g_{4}^{1}$ on the curve. Thus we almost always have a unique $g_{4}^{1}$ which imposes two linear conditions on $g_{p-1}^{r}$. From this the existence of the various plane models follows easily.

As a bonus it turns out that one can often characterize the case when this $g_{4}^{1}$ is itself the orbit space of a noncyclic group of automorphisms of order four. This characterization is again in terms of the vanishing properties of the theta function at half-periods. So the investigation ends where it began with characterizing certain automorphism groups in terms of vanishing properties of the theta function.

In Part I of this paper we considered Riemann surfaces of genus $3 r$ admitting a simple half-canonical linear series of dimension $r$. In Part II we now consider Riemann surfaces of genus $3 r+2$ admitting two simple half-canonical linear series of dimension $r$. In Part III we hope to consider the case of genus $3 r+3$ where the Riemann surface admits four simple half-canonical linear series of dimension $r$ whose sum is bicanonical. 
In $\$ 2$ of this paper we will summarize the necessary preliminary material. In $\$ 3$ we will consider the general case $r \geqslant 4$, and we leave the cases $r=1,2$, and 3 to $\$ 4$ where more special techniques appear to be necessary. In $\$ 5$ we consider when the $g_{4}^{1}$ is the set of orbits for a noncyclic group of automorphisms of order four. Finally, we have included an appendix which completes the discussion of automorphisms in Part I.

All results will be stated in terms of half-canonical linear series. We will omit the translation via Riemann's vanishing theorem to statements about the vanishing of the theta function of half-periods. An example of this type of translation will be found in [3].

2. Notation, definitions, and preliminary results. Let $W_{p}$ be a closed Riemann surface of genus $p$. A divisor $D=n_{1} z_{1}+n_{2} z_{2}+\cdots+n_{s} z_{s}$ is a zero chain on $W_{p}$ where the $n_{i}$ are integers and the $z_{i}$ are points on $W_{p}$. A divisor will be called integral $(D \geqslant 0)$ if each $n_{i} \geqslant 0$. If $f$ is a meromorphic function on $W_{p}$ then its divisor, $(f)$, can be written: $(f)=D_{1}-D_{2}$; where $D_{1}$ is the divisor of zeros of $f$ and $D_{2}$ is the divisor of poles. We say two divisors $D_{1}$ and $D_{2}$ (not necessarily integral) are (linearly) equivalent if there is a (meromorphic) function $f$ so that $(f)=D_{1}-D_{2}$. If $D$ is a divisor we say that a function $f$ is a multiple of $D$ if $(f)+D \geqslant 0$. The set of multiples of $D$ is a finite dimensional vector space. A linear series of degree $n$ and dimension $r$, written $g_{n}^{r}$, is a set of integral divisors $\{(f)+D\}$ where $D$ is an integral divisor of degree $n$ and $f$ ranges over an $r+1$ dimensional subspace of the multiples of $D$. Such a linear series will be called complete if the multiples of $D$ have dimension $r+1$. In such a case we write $|D|=g_{n}^{r}$; that is, $g_{n}^{r}=\left\{D^{\prime} \geqslant 0: D^{\prime} \equiv D\right\}$. For example, the canonical series $g_{2 p-2}^{p-1}$ is equal to $|K|$ where $K$ is the set of zeros of an abelian differential of the first kind.

The Riemann-Roch theorem asserts that for a complete $g_{n}^{r}, r=n-p+i$ where $i$ is the index of specialty ${ }^{2}$ of any divisor in $g_{n}^{r}$. An integral divisor will be called special if it is part of an integral canonical divisor. If $g_{n}^{r}+g_{n^{\prime}}^{r^{\prime}}$ is the canonical series then the Brill-Noether form of the Riemann-Roch theorem asserts that $n-2 r=n^{\prime}$ $-2 r^{\prime}$. Clifford's theorem asserts that in this case $n-2 r$ is nonnegative and is zero if and only if $n=0, n=2 p-2$, or $W_{p}$ is hyperelliptic.

For two integral divisors $D_{1}$ and $D_{2},\left(D_{1}, D_{2}\right)$ will denote their greatest common divisor. We will say that $D_{1}$ and $D_{2}$ are disjoint if $\left(D_{1}, D_{2}\right)=0$.

In this paper we will use the following terms. A pair will be an integral divisor of degree two and a triple will be an integral divisor of degree three. The points of a pair or triple need not be distinct. Finally, we will of ten write " $D \equiv g_{n}^{r}$ " for " $|D|=g_{n}^{r "}$ where there is no danger of confusion.

Linear series may be complete or incomplete, with or without fixed points, and simple or composite. If $g_{n}^{r}$ is a linear series on $W_{p}$ then $g_{n}^{r}-x$ will denote the linear

\footnotetext{
${ }^{2}$ The index of specialty of a divisor $D$ is the dimension of the space of holomorphic differentials whose divisors contain $D$.
} 
series of degree $n-1$ of divisors of $g_{n}^{r}$ passing through $x$ not counting $x$. If $x$ is not a fixed point of $g_{n}^{r}$ then $g_{n}^{r}-x=g_{n-1}^{r-1}$.

A linear series $g_{n}^{r}(r \geqslant 2)$ is simple if, for a general choice of $x$ on $W_{p}, g_{n}^{r}-x$ has the same fixed points as $g_{n}^{r}$. In this situation for $r \geqslant 3$ and a general choice of $x$, $g_{n}^{r}-x$ is also simple. If $g_{n}^{r}$ is simple and without fixed points then $W_{p}$ can be realized as a curve $C_{n}$ in $P^{r}$, and the hyperplane sections of the curve cut out the $g_{n}^{r}$. By a $k$-point of $g_{n}^{r}$ we mean an integral divisor of degree $k, x_{1}+\cdots+x_{k}$, so that whenever a divisor of $g_{n}^{r}$ contains one of the $x$ 's, it contains all $k$ of them, but not necessarily any further point of $W_{p}$. On the corresponding projective curve $C_{n}$ this corresponds to a singularity of multiplicity $k$, which we will also call a $k$-point of $C_{n}$.

A linear series $g_{n}^{r}$ is composite if, for every choice of $x$ on $W_{p}, g_{n}^{r}-x$ has more fixed points than $g_{n}^{r}$. In this case $W_{p}$ is a $t$-sheeted covering of a surface $W_{q}$, and a divisor of nonfixed points of $g_{n}^{r}$ is a union of fibers of the map $\phi: W_{p} \rightarrow W_{q}$. In such a case $W_{q}$ admits a $g_{(n-f) / t}^{r}$ which lifts via $\phi$ to $g_{n-f}^{r}$, the nonfixed points of $g_{n}^{r}$. If $x$ is not a fixed point of $g_{n}^{r}$ then $g_{n}^{r}-x$ has $t-1$ additional fixed points, the other points in the fiber of $\phi$ containing $x$. If $g_{n}^{r}$ is complete on $W_{p}$, so is $g_{(n-f) / t}^{r}$ on $W_{q}$.

If $g_{n}^{r}$ is a linear series an integral divisor $E=x_{1}+\cdots+x_{m}$ is said to impose $t$ (linear) conditions on $g_{n}^{r}$ if there are $t$ points of $E$, say $x_{1}+\cdots+x_{t}$ (not necessarily distinct) so that $g_{n}^{r}-\left(x_{1}+\cdots+x_{t}\right)$ has dimension $r-t$ and has $x_{t+1}+\cdots+x_{m}$ among its fixed points. It follows that if the first $t x$ 's are distinct points of $W_{p}$ then for each $k$ from 1 to $t$ there is a divisor of $g_{n}^{r}$ containing all the $x_{i}$, $i=1,2, \ldots, t$, except $x_{k}$.

If $g_{n}^{r}$ is a linear series and $g_{m}^{s}$ is another we say that $g_{m}^{s}$ imposes $t$ (linear) conditions on $g_{n}^{r}$ if one (and therefore every) divisor in $g_{m}^{s}$ imposes $t$ conditions on $g_{n}^{r}$. If $g_{n}^{r}$ is complete this means that there is a complete $g_{n-m}^{r-t}$ so that $g_{n}^{r} \equiv g_{m}^{s}+$ $g_{n-m}^{r-t}$. In particular, if a $g_{m}^{1}$ imposes one condition on $g_{n}^{r}$ then $g_{n}^{r}$ is composite and $g_{n}^{r} \equiv r g_{m}^{1}+D_{n-r m}$ where $D_{n-r m}$ is the divisor of fixed points of $g_{n}^{r}$.

We will use the following corollary of the Riemann-Roch theorem. $\mathrm{A} g_{n}^{1}(n<p)$ without fixed points imposes $n-1$ conditions on the canonical series and so imposes at most $n-1$ conditions on any special linear series. A simple special $g_{m}^{s}$ without fixed points imposes at most $m-s$ conditions on any other special linear series whose dimension is at least $m-s$.

If $W_{p}$ admits a simple $g_{n}^{2}$ without fixed points then $W_{p}$ admits a plane model of degree $n, C_{n}$. If $d$ is the number of double points suitably counted then

$$
p=(n-1)(n-2) / 2-d .
$$

To compute the dimension $R$ of all plane curves of degree $n$ with $s$ fixed ordinary singularities of multiplicities $k_{1}, k_{2}, \ldots, k_{s}$ we use the formula

$$
R=n(n+3) / 2-\sum_{k=1}^{s} k_{j}\left(k_{j}+1\right) / 2+\varepsilon
$$

where $\varepsilon \geqslant 0$. If we allow the singularities to vary in $P^{2}$ then the variety of such curves has dimension at least $R+2 s$. If one wishes to consider the Riemann 
surfaces corresponding to such a family then in computing the dimension of such Riemann surfaces in Teichmüller space one must subtract, at least, eight for the plane collineations.

For plane curves we shall have occasion to consider standard quadratic transformations with three fundamental points. If these points are $(1,0,0),(0,1,0)$ and $(0,0,1)$ then $x_{i} \rightarrow x_{j} x_{k},\{i, j, k\}=\{1,2,3\}$ gives the transformation [14, p. 74].

If $W_{p}$ admits a simple $g_{n}^{2}$ then formula (2.1) assures us that

$$
p \leqslant(n-1)(n-2) / 2 \text {. }
$$

The following inequality, due to Castelnuovo, generalizes this result.

2.1. TheOREM ([7], [8], [9]). Let $W_{p}$ admit a simple $g_{n}^{r}, r \geqslant 3$. Then

$$
p \leqslant(n-r+\varepsilon)(n-1-\varepsilon) / 2(r-1)
$$

where $0 \leqslant \varepsilon \leqslant r-1$ and $n-r+\varepsilon \equiv 0(\bmod r-1)$. If we have equality in formula (2.3), $n>2 r$, and $r \neq 5$, then $C_{n}$, the corresponding curve in $P^{r}$, lies on a rational normal scroll whose rulings cut out a $g_{m}^{1}$ on $W_{p}$ which imposes two conditions on $g_{n}^{r}$.

The case of most interest in this paper is when $W_{p}$ admits a simple $g_{3 r-1}^{r}$ and $p=3 r$. In that case $2 g_{3 r-1}^{r}$ is canonical, we have equality in formula (2.3), and $W_{3 r}$ admits a $g_{4}^{1}$ which imposes two conditions on $g_{3 r-1}^{r}[6$, Lemma 3.1].

As a consequence of the methods used by Castelnuovo the following results can be derived [5, Lemma 4.2]. They give lower bounds on the dimensions of the sums of certain linear series.

2.2. LemMa. Suppose $p \neq 0$.

(i) Suppose $g_{n}^{r}$ and $g_{m}^{s}$ are different simple linear series without fixed points, $n \geqslant r+s$, and $r \geqslant s$. Then $g_{n}^{r}+g_{m}^{s}=g_{n+m}^{r+2 s+e}$ where $\varepsilon \geqslant 0$.

(ii) Suppose $g_{n}^{r}$ is simple. Then $g_{n}^{r}+t g_{m}^{1}=g_{n+t m}^{r+2 t+\varepsilon}$ where $\varepsilon \geqslant 0$.

(iii) Suppose for $g_{n}^{1}$ and $g_{m}^{1}$ the two coverings of $P^{1}$ determined by these linear series do not admit a common factoring. Suppose $n \geqslant t+1$. Then $s g_{n}^{1}+t g_{m}^{1}=g_{s n+t m}^{t+t s+e}$ where $\varepsilon \geqslant 0$.

On $W_{p}$ a linear series $g_{p-1}^{r}$ is said to be half-canonical if the sum of any two divisors in $g_{p-1}^{r}$ is canonical; that is, $2 g_{p-1}^{r} \equiv g_{2 p-2}^{p-1}$. The following results are basic for half-canonical linear series.

2.3. Lemma [6, Lemma 2.4]. A $g_{m}^{1}$ without fixed points imposes at most $[m / 2]$ conditions on a half-canonical $g_{p-1}^{r}(m<2 r+1)$.

2.4. Theorem [6, Theorem 2.5]. Let $W_{p}$ admit a simple $g_{m}^{s}$ without fixed points and a half-canonical $g_{p-1}^{r}$ where $m-s \leqslant 2 r$. Then $g_{m}^{s}$ imposes at most $[(m-s+1) / 2]$ conditions on $g_{p-1}^{r}$. Also $g_{p-1}^{r}$ must be simple.

2.5. Corollary. $A g_{3}^{1}$ imposes one condition on a half-canonical $g_{p-1}^{r}$. A $g_{4}^{1}$ imposes at most two conditions on a half-canonical $g_{p-1}^{r}$.

Definition. A Riemann surface admitting a $g_{3}^{1}$ without fixed points will be called trigonal. 
Before continuing with results on half-canonical linear series we must briefly discuss automorphisms. By $A\left(W_{p}\right)$ we shall mean the finite group of automorphisms (conformal self-maps) of $W_{p}(p \geqslant 2)$. We shall be particularly interested in automorphisms of order two (involutions). A Riemann surface $W_{p}$ will be called $q$-hyperelliptic if $W_{p}$ admits an involution $T$ and the genus of $W_{p} /\langle T\rangle$ is at most $q$. Thus $P^{1}$, tori, and hyperelliptic Riemann surfaces are 0-hyperelliptic. 1-hyperelliptic surfaces which are not 0 -hyperelliptic are called elliptic-hyperelliptic. We have the following useful result concerning involutions and half-canonical linear series.

2.6. Lemma [6, Lemma 2.6]. Suppose $W_{p}$ is q-hyperelliptic and admits a simple half-canonical $g_{p-1}^{r}$. Then $r \leqslant q$.

We will also be interested in noncyclic groups of order four. If $W_{p}$ admits such a group and $T_{1}, T_{2}$, and $T_{3}$ are the involutions in the group $G$, let $p_{i}$ be the genus of $W_{p} /\left\langle T_{i}\right\rangle$ for $i=1,2$, and 3 , and let $p_{0}$ be the genus of $W_{p} / G$. Then $p+2 p_{0}=p_{1}$ $+p_{2}+p_{3}[1]$. Such a group $G$ on $W_{p}$ will be denoted by the symbol $\left(p ; p_{1}, p_{2}, p_{3}\right.$; $\left.p_{0}\right)$.

By using Lemma 2.6 we can obtain the following [6, Lemma 2.7].

2.7. Lemma. Let $W_{p}$ admit a simple half-canonical $g_{p-1}^{r}{ }^{3}$ Suppose $W_{p}$ admits a $g_{m}^{s}$ and $a g_{4}^{1}$ without fixed points so that $g_{4}^{1}$ imposes at most two conditions on $g_{m}^{s}$. Suppose finally that $s \leqslant r$ and $m-4 s<0$. Then $g_{m}^{s}$ is simple.

We will include a proof of the following lemma even though it is almost identical to that of the preceding lemma.

2.8. Lemma. Suppose $W_{p}$ admits a simple half-canonical $g_{p-1}^{r}$ and a $g_{4}^{1}$ without fixed points. Suppose $g_{4}^{1}$ imposes at most two conditions on another complete linear series $g_{m}^{s}$ where $m-2 s<2 r, s \geqslant 2$. Suppose finally that $g_{m}^{s}$ is composite. Then $g_{m}^{s}=s g_{4}^{1}+$ $D_{m-4 s}$ where $D_{m-4 s}$ is the divisor of fixed points of $g_{m}^{s}$.

Proof. Since $g_{m}^{s}$ is composite there is a $t$-sheeted covering $\phi: W_{p} \rightarrow W_{q}$ and a complete $g_{(m-f) / t}^{s}$ on $W_{q}$ which lifts to the nonfixed points of $g_{m}^{s}$. Since $g_{4}^{1}$ imposes at most two conditions on $g_{m}^{s}$ we see that on $W_{q}$ there is a $g_{4 / t}^{1}$ which lifts to $g_{4}^{1}$ and so $t=2$ or 4 . If $t=4$ then $W_{q}$ is $P^{1}$ and $g_{1}^{1}$ imposes one condition on $g_{(m-f) / 4}^{s}$. The result follows by lifting to $W_{p}$. If $t=2$ and $g_{(m-f) / 2}^{s}$ is special then $g_{2}^{1}$ imposes one condition on $g_{(m-f) / 2}^{s}$ and the result follows. If $g_{(m-f) / 2}^{s}$ is nonspecial then $q=$ $(m-f) / 2-s$ and $q \geqslant r$ by Lemma 2.6. This leads to the contradiction $m-2 s \geqslant$ 2r. Q.E.D.

The last lemma will be used constantly in the sequel.

2.9. Lemma. Suppose $W_{p}$ admits a $g_{4}^{1}$ without fixed points so that $2 g_{4}^{1}=g_{8}^{2}$ is complete. Suppose $h_{4}^{0}$ is complete and $2 h_{4}^{0} \equiv 2 g_{4}^{1}$. Then $h_{4}^{0}=P+Q$ where $P$ and $Q$ are disjoint pairs satisfying $2 P \equiv 2 Q \equiv g_{4}^{1}$.

\footnotetext{
${ }^{3}$ In [6, Lemma 2.7] the assumption that $p=3 r$ is unnecessary.
} 
Proof. $2 h_{4}^{0}$ contains points in at most only two divisors of $g_{4}^{1}$ and consequently, the same is true of $h_{4}^{0}$. But $h_{4}^{0}$ must contain points in at least two distinct divisors of $g_{4}^{1}$. Consequently, $h_{4}^{0}$ must contain points in precisely two distinct divisors of $g_{4}^{1}$. The result now follows (or [6, Lemma 2.8]). Q.E.D.

3. The cases $r \geqslant 4$. We now suppose that $W_{3 r+2}$ admits two half-canonical $g_{3 r+1}^{r}$ 's. We will prove the existence of certain plane models for $r \geqslant 4$ in this section, but some of the preliminary lemmas will be valid for smaller $r$. We first show that the $g_{3 r+1}^{r}$ 's must be simple.

3.1. Lemma. Suppose $r \geqslant 2$ and $W_{3 r+2}$ admits a composite half-canonical $g_{3 r+1}^{r}$. Then either $g_{3 r+1}^{r}$ is unique or $W_{3 r+2}$ admits at least 16 half-canonical $g_{3 r+1}^{r}$ 's. Thus if $W_{3 r+2}$ admits $2,3, \ldots, 14$, or 15 half-canonical $g_{3 r+1}^{r}$ 's they must all be simple.

Proof. Since $g_{3 r+1}^{r}$ is composite there is a $t$-sheeted covering $\phi: W_{p} \rightarrow W_{q}$ and a complete simple $g_{(3 r+1-f) / t}^{r}$ on $W_{q}$ which lifts to the nonfixed points of $g_{3 r+1}^{r}$. $(f$ is the number of fixed points of $g_{3 r+1}^{r}$.) If $t \geqslant 4$, since $(3 r+1-f) / t \geqslant r$ we have $1-f \geqslant r$, a contradiction. If $t=3$ then $1-f \geqslant 0$; consequently, $f=1, W_{q}$ admits a $g_{r}^{r}$, and $q=0$. $W_{p}$ admits a $g_{3}^{1}$ and $g_{3 r+1}^{r}=r g_{3}^{1}+x$ where $x$ is the fixed point. By Corollary 2.5 all half-canonical linear series are now composite. If $h_{3 r+1}^{r}$ is a second half-canonical linear series then $h_{3 r+1}^{r}=r g_{3}^{1}+y$. Since $2 g_{3 r+1}^{r} \equiv$ $2 h_{3 r+1}^{r}$ it follows that $2 x \equiv 2 y$, a contradiction. Consequently, if $t=3$ then the half-canonical $g_{3 r+1}^{r}$ is unique.

Now suppose $t=2$. If $g_{(3 r+1-f) / 2}^{r}$ is special then by Clifford's theorem we arrive at the contradiction $(3 r+1-f) / 2 \geqslant 2 r$. Consequently $g_{(3 r+1-f) / 2}^{r}$ is not special and is complete, so that $q=(r+1-f) / 2$ by the Riemann-Roch theorem. Thus $W_{p}$ is $q$-hyperelliptic where $p=3 r+2$ and $q=(r+1-f) / 2$. By the results of [4, p. 51] this implies that $W_{p}$ has vanishing properties for the theta function like a hyperelliptic Riemann surface of genus $p-2 q=2 r+1+f$. Moreover $W_{p}$ admits $2^{2 q}$ sets of such vanishing properties, one for each half-period in the Jacobian of $W_{q}$. Thus if $r$ is odd and $f=0$ then $W_{p}$ admits $2^{r+1}\left(=2^{2 q}\right)$ half-canonical $g_{3 r+1}^{r}$ 's. In general it can be shown that $W_{p}$ admits $2^{r-f+1}\left({ }^{4 r+2 f+4}\right)$ half-canonical $g_{3 r+1}^{r}$ 's. Since $f \geqslant 1$ when $r$ is even, the smallest value of this expression is $16(r=3$, $f=0){ }^{4} \quad$ Q.E.D.

3.2. Lemma. Suppose $r \geqslant 2$ and $W_{3 r+2}$ admits two simple half-canonical linear series $g_{3 r+1}^{r}$ and $h_{3 r+1}^{r}$. Then neither has a fixed point.

Proof. If $g_{3 r+1}^{r}=g_{3 r-1}^{r}+x+y$ where $x$ and $y$ are fixed points then $g_{3 r-1}^{r}$ is composite by Castelnuovo's inequality, Theorem 2.1.

If $g_{3 r+1}^{r}=g_{3 r}^{r}+x$ then by Lemma 2.2(i) we see that $g_{3 r}^{r}+h_{3 r+1}^{r}=g_{6 r+1}^{3 r+e}$ where $\varepsilon \geqslant 0$. By the Riemann-Roch theorem it follows that this latter series is special and so there is a point $y$ so that $g_{3 r}^{r}+h_{3 r+1}^{r}+y \equiv K$, a canonical divisor. Since $h_{3 r+1}^{r}$ is half-canonical it follows that $h_{3 r+1}^{r}=g_{3 r}^{r}+y$. Thus $K \equiv 2\left(g_{3 r}^{r}+x\right) \equiv 2\left(g_{3 r}^{r}+y\right)$, or $2 x \equiv 2 y$. This contradiction completes the proof. Q.E.D.

\footnotetext{
${ }^{4}$ In fact, if $f>2$ then $W_{p}$ admits half-canonical $g_{p-1}^{r+1}$ 's.
} 
3.3. LEMMA. If $r \geqslant 3$ and $W_{3 r+2}$ admits two simple half-canonical linear series of dimension $r$ then $W_{3 r+2}$ admits a $g_{4}^{1}$ without fixed points.

Proof. By the methods of $[4$, pp. 67, 68, 74] there is a smooth, two-sheeted cover of $W_{3 r+2}$ of genus $6 r+3$ and $g_{3 r+1}^{r}$ and $h_{3 r+1}^{r}$ lift to this cover to become equivalent. On $W_{6 r+3}$ they determine a half-canonical $g_{6 r+2}^{2 r+1}$. By the discussion following Theorem 2.1, $W_{6 r+3}$ admits a $g_{4}^{1}$ and consequently $W_{3 r+1}$ does also. That it is without fixed points follows from Corollary 2.5. Q.E.D.

Until further notice we will assume that $W_{3 r+2}$ admits two simple half-canonical linear series $g_{3 r+1}^{r}$ and $h_{3 r+1}^{r}$ and also a $g_{4}^{1}$ without fixed points. Since $g_{4}^{1}$ imposes two conditions on $g_{3 r+1}^{r}$ and $h_{3 r+1}^{r}$ we have

$$
g_{3 r+1}^{r} \equiv g_{4}^{1}+g_{3 r-3}^{r-2} \text { and } h_{3 r+1}^{r} \equiv g_{4}^{1}+h_{3 r-3}^{r-2}
$$

where these equations define $g_{3 r-3}^{r-2}$ and $h_{3 r-3}^{r-2}$.

3.4. LEMMA. If $r \geqslant 6$ then $g_{3 r-3}^{r-2}$ and $h_{3 r-3}^{r-2}$ are both simple.

Proof. We use Lemma 2.7 of $\$ 2$. Since $g_{4}^{1}$ imposes at most two conditions on $g_{3 r-3}^{r-2}$ and $3 r-3<4(r-2)$ we see that $g_{3 r-3}^{r-2}$ is simple. Q.E.D.

3.5. LEMMA. If $r=4$ or 5 then either $g_{3 r-3}^{r-2}$ or $h_{3 r-3}^{r-2}$ is simple.

Proof. If $r=5$ then $g_{3 r-3}^{r-2}=g_{12}^{3}$. By Lemma 2.8 we see that if $g_{12}^{3}$ is composite it must be $3 g_{4}^{1}$. Since $h_{12}^{3} \neq 3 g_{4}^{1}$ in this case we see that $h_{12}^{3}$ must be simple.

If $r=4$ and both are composite we see by Lemma 2.8 that $g_{9}^{2}=2 g_{4}^{1}+x$ and $h_{9}^{2}=2 g_{4}^{1}+y$. Since $2 g_{9}^{2} \equiv 2 h_{9}^{2}$ we arrive at the contradiction $2 x \equiv 2 y$. Q.E.D.

3.6. LEMMA. If $r \geqslant 4$ and $g_{3 r-3}^{r-2}$ is simple, then $g_{3 r-3}^{r-2}$ is without fixed points.

Proof. If $g_{3 r-3}^{r-2}=g_{3 r-4}^{r-2}+x$ then $g_{3 r+1}^{r} \equiv g_{4}^{1}+g_{3 r-3}^{r-2}+x=g_{3 r}^{r+e}+x$ by Lemma 2.2(ii). We see that $\varepsilon=0$ and that $g_{3 r+1}^{r}$ has a fixed point. This contradicts Lemma 3.2. Q.E.D.

Definition. For a plane curve a $(2,4)$-singularity or simply a $(2,4)$-point will be a double point with a unique tangent line which has four intersections with the curve at the double point. This tangent will be called the $(2,4)$-tangent.

Examples of $(2,4)$-points are tacnodes and ramphoid cusps. [14, p. 56]. A $(2,4)$-point contributes at least two to the double points of the plane curve suitably counted.

For a simple $g_{n}^{r}$ a $(2,4)$-point is a 2-point $P$ which imposes one condition on $g_{n}^{r}$ and $2 P$ imposes two conditions on $g_{n}^{r}$.

3.7. THEOREM. Suppose $r \geqslant 4$ and $W_{3 r+2}$ admits precisely two half-canonical linear series $g_{3 r+1}^{r}$ and $h_{3 r+1}^{r}$. Then $W_{3 r+2}$ admits as a plane model a curve $C_{2 r+3}$ with $r-2$ 3-points, one $(2 r-1)$-point, and two $(2,4)$-points with both $(2,4)$-tangents passing through the $(2 r-1)$-point.

Proof. We know that both half-canonical series are simple and we can assume by Lemmas 3.4, 3.5, and 3.6 that $g_{3 r-3}^{r-2}$ is simple and without fixed points. By Theorem 2.4, $g_{3 r-3}^{r-2}$ imposes at most $r$ conditions on $h_{3 r+1}^{r}$. Thus there is a complete 
$h_{4}^{0}$ so that $h_{3 r+1}^{r} \equiv g_{3 r-3}^{r-2}+h_{4}^{0}$. Since $h_{3 r+1}^{r} \equiv h_{3 r-3}^{r-2}+g_{4}^{1}$ we have $K \equiv 2 h_{3 r+1}^{r} \equiv$ $h_{3 r-3}^{r-2}+g_{4}^{1}+g_{3 r-3}^{r-2}+h_{4}^{0}$. Since we have half-canonical series we see that

$$
\begin{aligned}
& h_{3 r+1}^{r} \equiv h_{4}^{0}+g_{3 r-3}^{r-2} \equiv h_{3 r-3}^{r-2}+g_{4}^{1}, \\
& g_{3 r+1}^{r} \equiv h_{4}^{0}+h_{3 r-3}^{r-2} \equiv g_{3 r-3}^{r-2}+g_{4}^{1} .
\end{aligned}
$$

It follows that $2 h_{4}^{0} \equiv 2 g_{4}^{1}$. Since $p \geqslant 14$ Theorem 2.1 assures us that any $g_{8}^{3}$ must be composite, and this is impossible. Consequently $2 g_{4}^{1}=g_{8}^{2}$ is complete. By Lemma 2.9 there are two pairs $P$ and $Q$ so that $h_{4}^{0}=P+Q$ and $2 P \equiv 2 Q \equiv g_{4}^{1}$ where $(P, Q)=0$.

Now choose on $W_{p} r-2$ points $x_{1}, \ldots, x_{r-2}$ generically so that they lie on different divisors, $x_{i}+T_{i}$, of $g_{4}^{1}$ and so that these divisors are disjoint from $P$ and $Q$. Now

$$
g_{3 r+1}^{r}-\left(x_{1}+\cdots+x_{r-2}\right)=g_{2 r+3}^{2}=g_{4}^{1}+D_{2 r-1}
$$

since $g_{4}^{1}$ still imposes two conditions on the simple $g_{2 r+3}^{2} \cdot g_{2 r+3}^{2}$ corresponds to a plane curve $C_{2 r+3}$. Each triple $T_{i}$ corresponds to a 3-point of $C_{2 r+3}$ and $D_{2 r-1}$ corresponds to a $(2 r-1)$-point.

We now show that $P$ and $Q$ determine $(2,4)$-points on $C_{2 r+3}$.

$$
\begin{aligned}
g_{2 r+3}^{2} & =g_{3 r+1}^{r}-\left(x_{1}+\cdots+x_{r-2}\right) \\
& =h_{4}^{0}+h_{3 r-3}^{r-2}-\left(x_{1}+\cdots+x_{r-2}\right) \\
& =P+Q+E_{2 r-1}=2 P+D_{2 r-1} \quad\left(2 P \equiv g_{4}^{1}\right)
\end{aligned}
$$

where $E_{2 r-1}$ is some divisor on $W_{p}$ of degree $2 r-1$. By the choice of the $x$ 's we can assume that $\left(E_{2 r-1}, P\right)=0$ so that the last two divisors in the above formulas are not the same. Thus the pair $P$ lies on two distinct divisors of $g_{2 r+3}^{2}$ and so is a double point. That it is a $(2,4)$-point follows from the form of the last divisor above and we see that the $(2,4)$-tangent cuts out $2 P+D_{2 r-1}$.

For the double points of $C_{2 r+3}$ (suitably counted) the $T_{i}$ 's contribute at least $3(r-2), D_{2 r-1}$ contributes at least $(2 r-1)(2 r-2) / 2$, and $P$ and $Q$ contribute at least 4. By formula (2.1) we have

$$
\begin{aligned}
p= & 3 r+2 \leqslant(2 r+2)(2 r+1) / 2-3(r-2) \\
& -(2 r-1)(2 r-2) / 2-4 .
\end{aligned}
$$

But the right-hand side of this last formula is $3 r+2$ so that each of the $r+1$ singularities contributes the minimum possible to the double points of $C_{2 r+3}$ suitably counted. Q.E.D.

If $r=4$ or $5, h_{3 r-3}^{r-2}$ can be composite. This will occur when the $T$ 's and $P$ and $Q$ are collinear in $P^{2}$.

3.8. Corollary. If $r \geqslant 4, W_{3 r+2}$ can admit at most two simple half-canonical $g_{3 r+1}^{r} s$.

Proof. Suppose $k_{3 r+1}^{r}$ is a third simple half-canonical series. As above, there is a complete $k_{4}^{0}$ so that $k_{3 r+1}^{r} \equiv g_{3 r-3}^{r-2}+k_{4}^{0}, 2 k_{4}^{0} \equiv 2 g_{4}^{1}$ and $k_{4}^{0}=R+S$ where $R$ and $S$ are pairs. Since $k_{4}^{0} \neq h_{4}^{0}$ the proof of Theorem 3.7 shows that $C_{2 r+3}$ admits a third $(2,4)$-point which will add another two to the double points suitably counted. Since 
we already have equality in formula (3.3) this is impossible. Thus the existence of $k_{3 r+1}^{r}$ is impossible. Q.E.D.

By the same argument we have the following corollary.

3.9. Corollary. Let $R$ be a pair so that $R$ imposes one condition on $g_{3 r+1}^{r}$. Then $R=P$ or $R=Q$.

Proof. $R$ would add at least one to the double points of $C_{2 r+3}$ suitably counted. This is one too many unless $R=P$ or $Q$. Q.E.D.

If all the singularities are in general position in $P^{2}$ we can perform quadratic transformations to obtain the models of [2, p. 18]. First perform a standard quadratic transformation with fundamental points $P, Q$ and one of the 3-points. This gives a curve $C_{4 r-1}$ with $r-3$ 3-points and six further singularities at the pairs of opposite vertices of a complete quadrilateral of multiplicities 2, 2, 2r$2,2 r-2,2 r-1,2 r-1$. Now perform a quadratic transformation with the two singularities of multiplicities $2 r-1$ and one 3-point as fundamental points. One obtains a $C_{4 r-3}$ with singularities of order $2,2,2 r-3,2 r-3,2 r-2,2 r-2$, and $r-4$ 3-points. One proceeds with $r-4$ similar quadratic transformations to obtain $C_{2 r+5}$ with precisely six singularities of multiplicities $2,2, r+1, r+1, r+$ 2 , and $r+2$ and no 3-points. Singularities of the same multiplicity occur at opposite vertices of a complete quadrilateral.

To obtain the models of $[2$, p. 21] for $V=1$ we now perform a quadratic transformation on $C_{2 r+5}$ with fundamental points at singularities of multiplicities $r+2, r+2$, and $r+1$. We obtain a curve $C_{r+5}$ with an $(r+1)$-point $R$ and two $(2,4)$-points with the $(2,4)$ tangents passing through $R$. For $r$ even we could obtain the same model by the formula

$$
g_{3 r+1}^{r}-((r-2) / 2) g_{4}^{1}=g_{r+5}^{2} .
$$

These latter plane models allow us to compute the dimension in Teichmüller space of the family of Riemann surfaces of genus $3 r+2$ admitting two simple half-canonical $g_{3 r+1}^{r}$ 's, since the models are almost unique. The dimension is $5 r+5$ which is equal to $(r+5)(r+8) / 2-(r+1)(r+2) / 2-6-6-2$. We subtract 6 for each $(2,4)$-point (which is a tacnode in general) since the direction of the tangent is prescribed. We also subtract two for the two dimensional family of collineations of $P^{2}$ which leave the three singularities fixed.

4. The cases $r=3,2$, and 1 . We now consider the cases not covered in $\S 3$.

$r=3$. $W_{11}$ admits two half-canonical linear series $g_{10}^{3}$ and $h_{10}^{3}$ which are simple and without fixed points (Lemmas 3.1, 3.2), and $W_{11}$ also admits a unique $g_{4}^{1}$ without fixed points (Lemma 3.3). Consequently we can write

$$
g_{10}^{3} \equiv g_{4}^{1}+g_{6}^{1}, \quad h_{10}^{3} \equiv g_{4}^{1}+h_{6}^{1} .
$$

4.1. LEMMA. If $g_{6}^{1}$ has a fixed point it must have two fixed points.

Proof. If $g_{6}^{1}=g_{5}^{1}+x$ then $g_{10}^{3} \equiv g_{4}^{1}+g_{5}^{1}+x \equiv g_{9}^{3+e}+x$ by Lemma 2.2(iii). Consequently $\varepsilon=0$ and $g_{10}^{3}$ has a fixed point. This is a contradiction. Q.E.D.

We distinguish three cases: 
(i) $g_{6}^{1}$ and $h_{6}^{1}$ are both without fixed points.

(ii) $g_{6}^{1}$ is without fixed points, $h_{6}^{1}=g_{4}^{1}+R$ where $R$ is a pair.

(iii) $g_{6}^{1}=g_{4}^{1}+P\left(g_{10}^{3} \equiv 2 g_{4}^{1}+P\right), h_{6}^{1}=g_{4}^{1}+Q\left(h_{10}^{3} \equiv 2 g_{4}^{1}+Q\right)$

where $P$ and $Q$ are pairs so that $2 P \equiv 2 Q \equiv g_{4}^{1}$ and $(P, Q)=0$.

4.2. Lemma. There is a divisor $h_{4}^{0}=P+Q$ where $2 P \equiv 2 Q \equiv g_{4}^{1}$. Also

$$
g_{10}^{3} \equiv g_{4}^{1}+g_{6}^{1} \equiv h_{4}^{0}+h_{6}^{1}, \quad h_{10}^{3} \equiv g_{4}^{1}+h_{6}^{1} \equiv h_{4}^{0}+g_{6}^{1} \text {. }
$$

Proof. Case (iii). Define $P$ and $Q$ by the equations used in describing case (iii). Then

$$
h_{4}^{0}+h_{6}^{1}=P+Q+Q+g_{4}^{1}=P+g_{4}^{1}+g_{4}^{1}=g_{10}^{3}
$$

and similarly for $h_{10}^{3}$.

Cases (i) and (ii). Since $g_{6}^{1}$ is without fixed points define $h_{4}^{0}$ by $h_{4}^{0}=h_{10}^{3}-g_{6}^{1}$. Then

$$
K \equiv 2 h_{10}^{3} \equiv h_{4}^{0}+g_{6}^{1}+g_{4}^{1}+h_{6}^{1} .
$$

Since we are dealing with half-canonical divisors formula (4.1) follows. Since $2 h_{4}^{0}=2 g_{4}^{1}$ the proof is completed by Lemma 2.9. Q.E.D.

Now in all cases $g_{10}^{3} \equiv 2 P+g_{6}^{1} \equiv 2 Q+g_{6}^{1} \equiv P+Q+h_{6}^{1}$, so that $g_{10}^{3}-Q \equiv$ $Q+g_{6}^{1} \equiv P+h_{6}^{1} \equiv h_{10}^{3}-P \equiv g_{8}^{s}$ where $s=1$ or 2 . In cases (i) and (ii) it is easy to see that $s=2$, but it is not so obvious in case (iii), so we proceed as follows.

Consider $g_{10}^{3}+Q \equiv 2 Q+g_{8}^{s} \equiv g_{4}^{1}+g_{8}^{s} \equiv g_{12}^{t}$. Now $g_{10}^{3}+Q \equiv 2 P+g_{8}^{s} \equiv h_{10}^{3}$ $+P$ and $(P, Q)=0$, so we see that $g_{12}^{t}$ is without fixed points. Also $g_{12}^{t}+g_{8}^{s} \equiv K$ and so $t=s+2$. But if $s=1$ then $t=3$ and $g_{12}^{t}$ has fixed points; consequently $s=2$. ( $s=3$ is impossible.) Moreover, since $g_{4}^{1}+g_{8}^{2} \equiv g_{12}^{4}$ we see that $g_{4}^{1}$ imposes at most two conditions on $g_{8}^{2}$. By Lemma 2.8 it follows that $g_{8}^{2}$ is simple since $g_{8}^{2} \neq 2 g_{4}^{1}$.

The plane curve $C_{8}$ corresponding to $g_{8}^{2}$ has a 4-point $D_{4}$ since $g_{4}^{1}$ imposes two conditions on $g_{8}^{2}$. Consequently, we have

$$
g_{8}^{2} \equiv Q+g_{6}^{1} \equiv P+h_{6}^{1} \equiv D_{4}+g_{4}^{1}
$$

In case (i) the divisors $P, Q$, and $D_{4}$ are pairwise disjoint. In case (ii) $D_{4}=R+Q$ and in case (iii) $D_{4}=P+Q$. In any case $P$ and $Q$ contribute at least two and $D_{4}$ contributes at least six to the double points suitably counted. Since ten is the maximum for the double points suitably counted, we see that $P, Q$, and $D_{4}$ contribute precisely the amounts indicated. Thus for $C_{8}$ cases (i), (ii), and (iii) correspond to none, one, or both of $P$ and $Q$ being in the first neighborhood of $D_{4}$. In all cases the $(2,4)$-tangents at $P$ and $Q$ pass through $D_{4}$.

4.3. TheOREM. Let $W_{11}$ admit two simple half-canonical linear series. Then $W_{11}$ admits a plane model $C_{8}$ with a 4-point and two $(2,4)$-points with the $(2,4)$-tangents passing through the 4-point. One or both of the $(2,4)$-points may be in the first neighborhood of the 4-point.

Again by a careful examination of the proof we see that Corollary 3.8 follows for $r=3$. 
4.4. Corollary. $W_{11}$ cannot admit three simple half-canonical $g_{10}^{3}$ 's.

$r=2$. Suppose $W_{8}$ admits two simple half-canonical linear series $g_{7}^{2}$ and $h_{7}^{2}$. We know that neither has a fixed point but an added argument is needed to show the existence of a $g_{4}^{1}$.

4.5. LEMMA. $W_{8}$ admits $a g_{4}^{1}$.

Proof. $W_{8}$ admits a $g_{5}^{1}$ or a $g_{4}^{1}$. If $W_{8}$ admits a $g_{5}^{1}$ then by Lemma 2.3 we have $g_{7}^{2} \equiv g_{5}^{1}+P$ and $h_{7}^{2} \equiv g_{5}^{1}+Q$ where $(P, Q)=0$. Then $2 P \equiv 2 Q$ and this gives a $g_{4}^{1}$.

4.6. LEMMA. $g_{4}^{1}$ is unique and $2 g_{4}^{1} \equiv g_{8}^{2}$ is complete.

Proof. First we show that $W_{8}$ does not admit a $g_{8}^{3}$. If it does then $K-g_{8}^{3} \equiv g_{6}^{2}$. If $g_{6}^{2}$ is simple then by Theorem $2.4 g_{7}^{2}=g_{6}^{2}+x$ and $h_{7}^{2}=h_{6}^{2}+y$ and $2 x \equiv 2 y$, a contradiction. If $g_{6}^{2}$ is composite then $W_{7}$ is trigonal or 1-hyperelliptic, both possibilities being contradictions.

If $W_{p}$ admits a $g_{4}^{1}$ and an $h_{4}^{1}$ then $g_{4}^{1}+h_{4}^{1} \equiv g_{8}^{3}$, a contradiction. Moreover $2 g_{4}^{1} \equiv g_{8}^{2+\varepsilon}$ and $\varepsilon$ must be 0 . Q.E.D.

Now let $g_{7}^{2} \equiv g_{4}^{1}+g_{3}^{0}$ and $h_{7}^{2} \equiv g_{4}^{1}+h_{3}^{0}$. Then $g_{7}^{2}+h_{3}^{0} \equiv g_{3}^{0}+g_{4}^{1}+h_{3}^{0} \equiv g_{3}^{0}+$ $h_{7}^{2} \equiv g_{10}^{s}$ where $s \geqslant 3$ since $s=2$ is clearly impossible. Thus $g_{10}^{s}$ is special and there is a divisor $h_{4}^{0}$ so that

$$
K \equiv g_{3}^{0}+g_{4}^{1}+h_{3}^{0}+h_{4}^{0} .
$$

It follows that $g_{7}^{2}=h_{3}^{0}+h_{4}^{0}$ and $h_{7}^{2}=g_{3}^{0}+h_{4}^{0}$ and so $h_{4}^{0}$ has dimension zero. Moreover $2 h_{4}^{0} \equiv 2 g_{4}^{1}$. By Lemma $2.9 h_{4}^{0}=P+Q, 2 P \equiv 2 Q \equiv g_{4}^{1}$, and $(P, Q)=$ 0. Thus

$$
g_{7}^{2} \equiv g_{4}^{1}+g_{3}^{0} \equiv 2 P+g_{3}^{0} \equiv 2 Q+g_{3}^{0} \equiv P+Q+h_{3}^{0},
$$

$P$ and $Q$ are (2, 4)-points for $g_{7}^{2}$ and $g_{3}^{0}$ is a 3-point. Since $p=8<6 \cdot 5 / 2-3-2$ $-2=8$ this accounts for all the singularities of $g_{7}^{2}$. It is possible for $\left(P, g_{3}^{9}\right)$ or $\left(Q, g_{3}^{9}\right)$ to be nonzero but not both since $(P, Q)=0$. In this latter case one of the $(2,4)$-points is in the first neighborhood of $g_{3}^{0}$ on $C_{7}$, the plane curve corresponding to $g_{7}^{2}$.

4.7. TheORem. Suppose $W_{8}$ admits two simple half-canonical linear series. Then $W_{8}$ admits a plane model, $C_{7}$, with a 3-point and two $(2,4)$-points whose tangents pass through the 3-point. One but not both of the $(2,4)$-points may be in the first neighborhood of the 3-point.

Again we have the corollary:

4.8. CoROllaRY. $W_{8}$ cannot admit three simple half-canonical $g_{7}^{2} s$.

$r=1$. This case is covered in [3, p. 15]. However, there is an oversight in that discussion which should be mentioned. It is quite possible for the four doublepoints on the curve of degree seven to coalesce by pairs into two $(2,4)$-points and so the four lines in Figure 1 degenerate into three lines, one line being counted twice. 
5. Automorphisms. In this section we will disucss some possible automorphism groups of $W_{3 r+2}$ 's admitting two simple half-canonical $g_{3 r+1}^{r}$ 's, $r>2$.

Since $g_{4}^{1}$ is unique any automorphism must permute the divisors in $g_{4}^{1}$. If $N$ is the subgroup of $A\left(W_{3 r+2}\right)$ which leaves each divisor in $g_{4}^{1}$ fixed then $N$ is normal and $A\left(W_{3 r+2}\right) / N$ is isomorphic to a finite group of $P^{1}$. The order of $N$ is one, two, or four. Another normal subgroup is that one which leaves each half-canonical $g_{3 r+1}^{r}$ fixed. This subgroup will have index one or two since any automorphism permutes the two $g_{3 r+1}^{r}$ 's.

We shall devote the remainder of this section to considering the case where $g_{4}^{1}$ is the set of orbits of a noncyclic group of order four. As in $\$ 4$ of Part I, it turns out that such a four-group of automorphisms can be characterized by certain vanishing properties of the theta function: that is, by the existence of certain half-canonical $g_{3 r+1}^{r-1}$ 's.

To see this suppose that $W_{3 r+2}$ admits a noncyclic group of automorphisms of order four, $G$, whose orbits are $g_{4}^{1}$. Then each branched orbit is of the form $2 R$ where $R$ is a pair of two distinct points. By the Riemann-Hurwitz formula there are $3 r+5$ such pairs of which two are the $(2,4)$-points $P$ and $Q$. Thus there are $3 r+3$ other pairs $R_{1}, R_{2}, \ldots, R_{3 r+3}$. By formula (3.2) and the fact that $h_{4}^{0}=P+Q$ and $2 P \equiv 2 Q \equiv g_{4}^{1}$ we see that $g_{3 r+1}^{r}-P \equiv h_{3 r+1}^{r}-Q \equiv g_{3 r-1}^{r-1}$ and $g_{3 r+1}^{r}-Q \equiv$ $h_{3 r+1}^{r}-P \equiv h_{3 r-1}^{r-1}$. Now $g_{3 r-1}^{r-1}+R_{i}$ is half-canonical and complete as is $h_{3 r-1}^{r-1}+$ $R_{i}$. Let

$$
{ }_{i} g_{3 r+1}^{r-1}=g_{3 r-1}^{r-1}+R_{i} \text { and }{ }_{i} h_{3 r+1}^{r-1}=h_{3 r-1}^{r-1}+R_{i} .
$$

Then there are $3 r+3$ pairs $\left\{{ }_{i} g_{3 r+1}^{r-1},{ }_{i} h_{3 r+1}^{r-1}\right\}$.

We will now show that the existence of $3 r+3$ such pairs of half-canonical $g_{3 r+1}^{r-1}$ 's will insure that every branched divisor in $g_{4}^{1}$ is of the form $2 R$ where $R$ is a pair with two distinct points, at least for $r \geqslant 10$. The following lemma is the key.

5.1. Lemma. Suppose $W_{3 r+2}$ admits two half-canonical $g_{3 r+1}^{r}$ 's, $r>10$. If $W_{3 r+2}$ admits a half-canonical $g_{3 r+1}^{r-1}$ then $g_{3 r+1}^{r-1} \equiv g_{3 r+1}^{r}-P+R\left(\right.$ or $\left.g_{3 r+1}^{r}-Q+R\right)$ where $2 R \equiv g_{4}^{1}$.

Proof. We prove the lemma by assuming $g_{3 r+1}^{r-1}$ has precisely $t$ fixed points, $t=0,1,2, \ldots$, and showing that $t$ must equal 2 . This involves several cases.

Case (0). Assume that $g_{3 r+1}^{r-1}$ has no fixed points. By Lemma 2.7, $g_{3 r+1}^{r-1}$ is simple and $g_{3 r+1}^{r-1} \equiv g_{4}^{1}+g_{3 r-3}^{r-3}$ where again by Lemma $2.7, g_{3 r-3}^{r-3}$ is simple. $g_{3 r-3}^{r-3}$ is without fixed points and by Theorem 2.4 imposes at most $r$ conditions on $g_{3 r+1}^{r}$, so we have

$$
g_{3 r+1}^{r}=g_{3 r-3}^{r-3}+G_{4}^{0}
$$

where $2 G_{4}^{0} \equiv 2 g_{4}^{1}, G_{4}^{0}=R_{1}+R_{2}, 2 R_{1} \equiv 2 R_{2} \equiv g_{4}^{1}$, and $\left(R_{1}, R_{2}\right)=0$. Now $R_{1}+$ $R_{2}$ imposes three conditions on $g_{3 r+1}^{r}$ by formula (5.1) so one of the pairs, say $R_{1}$, imposes two conditions on $g_{3 r+1}^{r}$. But $2 R_{1} \equiv g_{4}^{1}$ which also imposes two conditions on $g_{3 r+1}^{r}$. Since $g_{3 r+1}^{r}-R_{1}=g_{3 r-3}^{r-3}+R_{2}$ we see that $R_{1}$ must be a fixed divisor of $g_{3 r-3}^{r-3}$, a contradiction.

Case (1). Assume that $g_{3 r+1}^{r-1}$ admits precisely one fixed point. $g_{3 r+1}^{r-1}=g_{3 r}^{r-1}+x$. Also $g_{3 r+1}^{r-1} \equiv g_{3 r-4}^{r-3}+g_{4}^{1}+x$ and $g_{3 r}^{r-1}$ and $g_{3 r-4}^{r-3}$ are simple by Lemma 2.7. Again 
$g_{3 r+1}^{r} \equiv g_{3 r-4}^{r-3}+G_{5}^{0}$ where $2 G_{5}^{0} \equiv 2 g_{4}^{1}+2 x \equiv g_{10}^{s}, s>2$.

If $s \geqslant 3$ then $g_{10}^{s}$ is simple and $p \leqslant 16$, a contradiction. Consequently $G_{5}^{0}=G_{4}^{0}$ $+x, 2 G_{4}^{0} \equiv 2 g_{4}^{1}, G_{4}^{0}=R_{1}+R_{2}, 2 R_{1} \equiv 2 R_{2} \equiv g_{4}^{1}$ and $\left(R_{1}, R_{2}\right)=0$. Thus

$$
g_{3 r+1}^{r} \equiv g_{3 r-4}^{r-3}+R_{1}+R_{2}+x .
$$

If either $R_{1}$ or $R_{2}$ imposes two conditions on $g_{3 r+1}^{r}$ we can argue as in case (0) to obtain a further fixed point of $g_{3 r-4}^{r-3}$ which is a contradiction. Consequently $R_{1}$ and $R_{2}$ must each impose one condition on $g_{3 r+1}^{r}$. By Corollary 3.9 this implies that $R_{1}=P$ and $R_{2}=Q$ (or vice versa). Consequently $g_{3 r+1}^{r} \equiv g_{3 r-4}^{r-3}+P+Q+x$. But exactly the same argument can be applied to $h_{3 r+1}^{r}$ and we arrive at the contradiction $g_{3 r+1}^{r}=h_{3 r+1}^{r}$.

Case (3). Assume that $g_{3 r+1}^{r-1}$ admits precisely three fixed points. $g_{3 r+1}^{r-1}=g_{3 r-2}^{r-1}+$ $T_{1}$ and $g_{3 r-2}^{r-1}$ imposes at most $r$ conditions on $g_{3 r+1}^{r}$. Thus $g_{3 r+1}^{r}=g_{3 r-2}^{r-1}+T_{2}$. It follows that $2 T_{1} \equiv 2 T_{2} \equiv g_{6}^{s}$ where $s=1$. Consequently $g_{6}^{1}=g_{4}^{1}+2 x$. Consequently $T_{2}=R+x$ where $2 R \equiv g_{4}^{1}$ and $R$ imposes one condition on $g_{3 r+1}^{r}$. This implies that $R=P$ or $Q$ and therefore that $g_{3 r+1}^{r}$ has a fixed point $x$, a contradiction.

Case (4). Assume that $g_{3 r+1}^{r-1}$ has precisely 4 fixed points, $g_{3 r+1}^{r-1}=g_{3 r-3}^{r-1}+G_{4}^{0}$. $g_{3 r-3}^{r-1}$ imposes at most $r-1$ conditions on $g_{3 r+1}^{r}$ so that $g_{3 r+1}^{r}=g_{3 r-3}^{r-1}+g_{4}^{1}$, contradicting the fact that $g_{4}^{1}$ imposes two conditions on $g_{3 r+1}^{r}$.

Case (5). Suppose $g_{3 r+1}^{r-1}$ has $5+k$ fixed points, $k \geqslant 0$. Then $g_{3 r+1}^{r-1}=g_{3 r-4-k}^{r-1}+$ $F$ where the degree of $F$ is $5+k$. By Castelnuovo's inequality $g_{3 r-4-k}^{r-1}$ is composite which contradicts Lemma 3.4 .

Case (2). $g_{3 r+1}^{r-1}$ must admit precisely two fixed points. If $g_{3 r+1}^{r-1}=g_{3 r-1}^{r-1}+R$ then $g_{3 r-1}^{r-1}$ imposes at most $r$ conditions on $g_{3 r+1}^{r}$, so $g_{3 r+1}^{r}=g_{3 r-1}^{r-1}+R^{\prime}$ where $2 R \equiv$ $2 R^{\prime} \equiv g_{4}^{1}$ and $R^{\prime}$ imposes one condition on $g_{3 r+1}^{r}$. By Corollary 3.9 it follows that $R^{\prime}=P$ or $Q$. If $g_{3 r+1}^{r} \equiv g_{3 r-1}^{r-1}+P$ and $h_{3 r+1}^{r} \equiv h_{3 r-1}^{r-1}+P$ then $h_{3 r-1}^{r-1}+R$ is a second half-canonical series of dimension $r-1$. Q.E.D.

We are now ready to complete the proof of the following theorem.

5.2. TheOREM. Suppose $W_{3 r+2}(r \geqslant 10)$ admits two simple half-canonical linear series $g_{3 r+1}^{r}$ and $h_{3 r+1}^{r}$. Then $g_{4}^{1}$ is the set of orbits of a noncyclic group of automorphisms of order four if and only if there are $3 r+3$ pairs of half-canonical linear series $\left\{{ }_{i} g_{3 r+1}^{r-1},{ }_{i} h_{3 r+1}^{r-1}\right\}$ so that $g_{3 r+1}^{r}+h_{3 r+1}^{r} \equiv{ }_{i} g_{3 r+1}^{r-1}+{ }_{i} h_{3 r+1}^{r-1}$ for all $i$.

Proof. Assuming the existence of the $3 r+3$ pairs of half-canonical linear series we infer the existence of $3 r+3$ pairs $R_{i}$ so that $2 R_{i} \equiv g_{4}^{1}$. Consequently we have $3 r+5$ pairs, $P, Q, R_{1}, R_{2}, \ldots, R_{3 r+3}$ whose doubles are in $g_{4}^{1}$. Each pair contributes at least two to the ramification of the four-sheeted covering of $P^{1}$ given by $g_{4}^{1}$. But the total ramification of this cover is $6 r+10$. Consequently each of the $3 r+5$ pairs must contribute precisely two to the ramification of this cover. This means that each pair must be two distinct points. Q.E.D.

Suppose $W_{3 r+2}$ admits such a four group $G$. Let $G_{1}, G_{2}$, and $G_{3}$ be the three subgroups of order two. By Lemma 2.6 the genus of $W_{3 r+2} / G_{i}$ must be $r$ or more. Since the sum of these three genera must be $3 r+2$, we see that $G$ is either a 
$(3 r+2 ; r+2, r, r ; 0)$ or a $(3 r+2 ; r+1, r+1, r ; 0)$. We shall presently give examples of the latter type.

But first let us heuristically count the dimension of the space of such $W_{3 r+2}$ 's. Since $W_{3 r+2}$ 's with two simple half-canonical linear series of dimension $r$ have dimension $5 r+5$, and the $g_{4}^{1}$ has two pairs $P$ and $Q$ so that $2 P \equiv 2 Q \equiv g_{4}^{1}$, it appears that the existence of the $3 r+3$ additional pairs $R_{i}$ imposes $3 r+3$ additional conditions. We would guess that $W_{3 r+2}$ 's with such a four group of automorphisms would have dimension $5 r+5-(3 r+3)=2 r+2$. In any event our examples will depend on $2 r+2$ parameters.

(Before proceeding to the examples the reader may wish to consult the Appendix where the analogous case of a $W_{3 r}$ admitting a simple $g_{3 r-1}^{r}$ is considered. Here the four group is a $(3 r ; r, r, r ; 0)$. The construction of this latter example is easier than that of the $(3 r+2 ; r+1, r+1, r ; 0)$.)

Now assume that $r$ is odd. Let $P_{1}(x)$ and $P_{2}(x)$ be polynomials of degree $r+1$ with $2 r+2$ distinct roots so that the Riemann surface $W_{r}$ of $\sqrt{P_{1} P_{2}}$ has genus $r$. Let $w=\sqrt{P_{1} / P_{2}}$. If $H$ is the hyperelliptic involution of $W_{r}$ then $H^{*} w=-w . w$ has order $r+1$ and is regular over $\infty$. Now fix a complex number $A$ and let $u=(A-w) /(A+w)$. Choose $A$ so that $u$ has no multiple zeros and poles. $u$ is a function of degree $r+1$ and $H^{*} u=1 / u$. If $D_{1}-D_{2}$ is the divisor of $u$ then $H D_{1}=D_{2}$. If $\phi: W_{r} \rightarrow P^{1}$ is the two-sheeted cover of $W_{r}$ over $P^{1}$, then it is easy to see that $\phi\left(D_{1}\right)\left(=\phi\left(D_{2}\right)\right)$ is the set of zeros of $P_{1}(x)-A^{2} P_{2}(x)$. Now let $d_{1}$ and $d_{2}$ be two different complex numbers which are not roots of $P_{1}, P_{2}$, or $P_{1}-A^{2} P_{2}$. Let $v=\left(x-d_{1}\right) u /\left(x-d_{2}\right)$, a function of order $r+3$ on $W_{r}$. Let $W_{3 r+2}$ be the two-sheeted cover of $W_{r}$ where $\sqrt{v}$ is singled valued. The covering $W_{3 r+2} \rightarrow W_{r}$ is branched above the zeros and poles of $w$ and also above $\phi^{-1}\left(d_{1}\right)$ and $\phi^{-1}\left(d_{2}\right)$. Thus the total ramification of this cover is $2 r+6$. On $W_{3 r+2}$ let $P$ be the two points above $\phi^{-1}\left(d_{1}\right)$, let $Q$ be the pair above $\phi^{-1}\left(d_{2}\right)$, let $D_{1}^{\prime}$ be the points above $D_{1}$ and let $D_{2}^{\prime}$ be the points above $D_{2}$. The divisor of $\sqrt{v}$ is $P+D_{1}^{\prime}-\left(Q+D_{2}^{\prime}\right)$. On $W_{r}$ the function $v_{1}=\left(x-d_{2}\right)^{2} v /\left(x-d_{1}\right)^{2}$ also has a square root on $W_{3 r+2}$ and $\left(\sqrt{v_{1}}\right)$ is $\left(Q+D_{1}^{\prime}\right)-\left(P+D_{2}^{\prime}\right)$. Let $g_{r+3}^{1}=\left|P+D_{1}^{\prime}\right|=\left|Q+D_{2}^{\prime}\right|$ and $h_{r+3}^{1}=\left|Q+D_{1}^{\prime}\right|$ $=\left|P+D_{2}^{\prime}\right|$.

Now $2 P \equiv 2 Q \equiv g_{4}^{1}$, where $g_{4}^{1}$ is the set of orbits for $G$, a noncyclic group of order four on $W_{3 r+2}$. Consequently, $2 g_{r+3}^{1} \equiv 2 h_{r+3}^{1}$. Also by Lemma 2.2(iii) we have

$$
((r-1) / 2) g_{4}^{1}+g_{r+3}^{1} \equiv g_{3 r+1}^{r+\varepsilon}
$$

where $g_{3 r+1}^{r+\varepsilon}$ is simple and so $\varepsilon=0$ by Castelnuovo's inequality. Consequently $g_{r+3}^{1}$ is complete. Similarly $((r-1) / 2) g_{4}^{1}+h_{r+3}^{1} \equiv h_{3 r+1}^{r}$. It follows that $2 g_{3 r+1}^{r} \equiv$ $2 h_{3 r+1}^{r}$.

It remains to show that $2 g_{3 r+1}^{r} \equiv(r-1) g_{4}^{1}+P+D_{1}^{\prime}+Q+D_{2}^{\prime}$ is canonical. On $W_{r}$ let $B$ be the $2 r+2$ branch points of $\phi: W_{r} \rightarrow P^{1}$. Then $B \equiv(r+1) g_{2}^{1}$. If $B^{\prime}$ is the lift of $B$ to $W_{3 r+2}$ then

$$
B^{\prime} \equiv(r+1) g_{4}^{1}
$$


But on $W_{3 r+2}$ the branched locus is $2 g_{4}^{1}+K$ where $K$ is the canonical divisor on $W_{3 r+2}$. That is

$$
K \equiv B^{\prime}+P+D_{1}^{\prime}+Q+D_{2}^{\prime}-2 g_{4}^{\prime}
$$

This together with formula (5.2) completes the demonstration.

We now state without proof the following.

5.3. Corollary. If $W_{3 r+2}$ admits two simple half-canonical linear series of dimension $r$ and a four group $G$ of automorphisms whose orbits are a $g_{4}^{1}$ then

(i) $r$ is odd,

(ii) $G$ is $a(3 r+2 ; r+1, r+1, r ; 0)$, and

(iii) $G$ arises exactly as in the above example.

The above examples depend on $2 r+2$ parameters as follows: $2 r-1$ for $W_{r}$; one for $A$ in the definition of $u$, and two for $d_{1}$ and $d_{2}$. The function field on $W_{3 r+2}$ is $\left(P_{3}=P_{1}-A^{2} P_{2}\right) C\left(x, \sqrt{P_{1} P_{2}}, \sqrt{P_{1}\left(x-d_{1}\right)\left(x-d_{2}\right) P_{3}}\right)$.

6. Appendix, an example with reference to Part I. For $r$ odd, we shall construct a $2 r$-dimensional family of $W_{3 r}$ 's admitting a $(3 r ; r, r, r ; 0)$ and a $g_{r+1}^{1}$ without fixed points. By the formula $g_{3 r-1}^{r}=g_{r+1}^{1}+((r-1) / 2) g_{4}^{1}$ ([6], or Lemma 2.2(iii)) this will insure that $W_{3 r}$ admits a $g_{3 r-1}^{r}$ which is simple and necessarily half-canonical.

We start with two polynomials $P_{1}(x)$ and $P_{2}(x)$ each of degree $r+1$ and all of whose $2 r+2$ zeros are distinct. Thus the Riemann surface of $\sqrt{P_{1} P_{2}}$ is a hyperelliptic surface $W_{r}$ of genus $r$. On this surface consider the function $w=\sqrt{P_{1} / P_{2}}$. If $H$ is the hyperelliptic involution of $W_{r}$ then $H^{*} w=-w$. Also $w$ is of order $r+1$ and is regular over $\infty$. Now fix a complex number $A$ so that the function $u=(A-w) /(A+w)$, again a function of degree $r+1$, has no multiple zeros or poles. Notice that $H^{*} u=1 / u$, so that if the divisor of $u$ is $D_{1}-D_{2}$ then $H D_{1}=D_{2}$. If $\phi: W_{r} \rightarrow P^{1}$ is the two-sheeted cover of $W_{r}$ over $P^{1}$ then it is easy to see that $\phi\left(D_{1}\right)\left(=\phi\left(D_{2}\right)\right)$ is the set of zeros of $P_{1}-A^{2} P_{2}$ (which we will call $P_{3}$ ). Now consider the two-sheeted cover of $W_{r}$ with branch points over $D_{1}+D_{2}$ where the function $\sqrt{u}$ is single valued. The ramification of this cover is $2 r+2$ so this cover, $W_{3 r}$, has genus $3 r$. $D_{1}$ and $D_{2}$ lift to divisors $D_{1}^{\prime}$ and $D_{2}^{\prime}$ and $D_{1}^{\prime}-D_{2}^{\prime}$ is the divisor of $\sqrt{u}$. Consequently, $\left|D_{1}^{\prime}\right|=g_{r+1}^{1}$, and $W_{3 r}$ is the desired Riemann surface. It depends on $2 r$ parameters, $2 r-1$ for the choice of $W_{r}$ and one for the choice of $A$ in the definition of $u$.

Since the main purpose of these examples is to show the characterization of Theorem 4.3, Part I, is not a vacuous statement, we will conclude with some corollaries without further proofs.

6.1. Corollary. If $W_{3 r}$ admits a simple $g_{3 r-1}^{r}$ and $a(3 r ; r, r, r ; 0)$ then:

(i) $r$ is odd, and

(ii) the group arises exactly as in the previous examples.

The function field for $W_{3 r}$ is $C\left(x, \sqrt{P_{1} P_{2}}, \sqrt{P_{1} P_{3}}\right)$. 


\section{REFERENCES}

1. R. D. M. Accola, Riemann surfaces with automorphism groups admitting partitions, Proc. Amer. Math. Soc. 21 (1969), 477-482.

2. Algebraic curves and half-canonical linear series, Discontinuous Groups and Riemann Surfaces, Ann. of Math. Studies, no. 79, Princeton Univ. Press, Princeton, N. J., 1974, pp. 13-22.

3. Some loci of Teichmüller space for genus five defined by vanishing theta nulls, in Contributions to Analysis, Academic Press, New York and London, 1974, pp. 11-18.

4. _ Riemann surfaces, theta functions and abelian automorphism groups, Lecture Notes in Math., vol. 483, Springer-Verlag, Berlin and New York, 1975.

5. __, On Castelnuovo's inequality for algebraic curves. I, Trans. Amer. Math. Soc. 251 (1979), 357-363.

6. __ Plane models for Riemann surfaces admitting certain half-canonical linear series. I (Proc. Conference on Riemann surfaces, June 1978).

7. G. Castelnuovo, Richerche di geometria sulle curve algebriche, Atti Accad. Sci. Torino 24 (1889).

8. __ Sur multipli du una serie lineare di gruppi di punti, etc., Rend. Circ. Mat. Palermo 7 (1893), 89-110.

9. J. L. Coolidge, Algebraic plane curves, Dover, Oxford, 1931.

10. H. M. Farkas, Automorphisms of compact Riemann surfaces and the vanishing of theta constants, Bull. Amer. Math. Soc. 73 (1967), 231-232.

11. L. Kraus, Note über ausgewohnliche special Gruppen auf algebraischen Kurven, Math. Ann. 15 (1880), 310.

12. H. H. Martens, On the varieties of special divisors on a curve. II, J. Reine Angew. Math. 233 (1968), 89-100.

13. B. Riemann, Gesammelte mathematische Werke, Dover, New York, 1953.

14. R. Walker, Algebraic curves, Princeton Math. Series, vol. 13, Princeton Univ. Press, Princeton, N. J., 1950.

15. H. Weber, Über Gewisse in der Theorie der Abel'schen Funktionen, auftretende Ausnahmfalle, Math. Ann. 13 (1878), 35-48.

Department of Mathematics, Brown University, Providence, Rhode Island 02912 